\title{
Reflexiones acerca de prácticas lúdicas en educación en derechos humanos: el juego cooperativo
}

\author{
Reflections on Playful Practices in Human Rights \\ Education: Cooperative Play
}

\author{
Evelyn Cerdas Agüero \\ Universidad Nacional \\ Heredia, Costa Rica \\ evelyncer@yahoo.com
}

\section{Recibido: 16/05/2018 Aceptado: 31/08/2018}

Resumen. El presente trabajo se centra en la educación en derechos humanos como un espacio de innovación pedagógico en la universidad, presenta parte de las experiencias vividas en un curso que ofrece el IDELA a la comunidad universitaria denominado Espacios Lúdicos para la Educación en Derechos Humanos. El objetivo es generar una reflexión con respecto a la importancia del juego en la educación en derechos humanos en el contexto universitario. Se realiza un abordaje descriptivo y un análisis de algunas de las experiencias vividas. Se concluye que la educación en derechos humanos debe ser un proceso centrado en prácticas innovadoras, vivenciales y lúdicas que sobrepasen las propuestas tradicionales y magistrales, en las cuales el estudiantado pueda crear, participar y aprender valores por medio de espacios participativos y socioafectivos en los que se reconozcan los derechos humanos como prácticas basadas en una forma de vida más solidaria y respetuosa.

Palabras clave: educación en derechos humanos, docencia, estudiantes, lúdica, juego.

Abstract: This work focuses on human rights education as a space for pedagogical innovation at the university. It presents part of the experiences lived in the course "Recreational Spaces for human rights education," offered by IDELA to the university community. This course aims to generate a reflection on the importance of playing in human rights education in the university context. A descriptive approach and an analysis of some of the lived experiences were implemented. It is concluded that human rights education should be a process centered on innovative, experiential, and playful practices that go beyond traditional and magisterial proposals, in which students can create, participate, and learn values through participatory and socio-affective spaces in which human rights are recognized as practices based on a more supportive and respectful way of life.

Keywords: education on human rights, teaching, students, play, games. 


\section{Introducción}

Educar en derechos humanos es una tarea compleja y posible que implica la vivencia, la participación, la visión crítica y la búsqueda colectiva de aprendizajes significativos que trascienden varios criterios pedagógicos tradicionales y confluyen en un entramado de prácticas participativas, afectivas, lúdicas y dialógicas.

En el presente trabajo se presenta una visión de la educación en derechos humanos en el contexto de un curso universitario como un aprendizaje en comunidad basado en la lúdica, donde se privilegia el juego cooperativo, el disfrute, la cooperación y la vivencia como bases fundamentales para el encuentro pedagógico.

De forma que en este abordaje se muestra parte de las vivencias y experiencias en el curso universitario Espacios Lúdicos para la Educación en Derechos Humanos, ofrecido por el Instituto de Estudios Latinoamericanos (IDELA) como curso optativo para todas las carreras en el Campus Omar Dengo de la Universidad Nacional, Costa Rica.

\section{El juego cooperativo en la educación en derechos humanos}

La educación en derechos humanos no es una educación teórica, sino una que prioriza la vivencia, la empatía, la afectividad y el aprendizaje significativo por medio de la reflexión y la experiencia en carne propia de situaciones reales, criticas que afectan a las personas, la realidad social y las interacciones humanas. Esta "tiene por objeto fomentar el entendimiento de que cada persona comparte la responsabilidad de lograr que los derechos humanos sean una realidad en cada comunidad y en la sociedad en su conjunto" (Asamblea General de Naciones Unidas, 2014, p. 3).

Se refiere así a una educación transformadora, por lo tanto, crítica y dinámica, así, en las formas de mediación pedagógica deberían privilegiarse las técnicas en las cuales se den procesos participativos, prácticos, vivenciales y dinámicos, los cuales el juego puede privilegiar de forma efectiva.

Esto porque la educación en derechos humanos se articula en torno a principios básicos que permiten a las personas desarrollar habilidades hacia formas de convivencia individuales y colectivas de forma más pacífica y respetuosa de los derechos humanos. Digo individuales porque me parece que esta educación se enfoca en el individuo como elemento principal de la convivencia y de la vida en sociedad, por lo que esta puede enfocarse inicialmente en un sujeto que es susceptible de ser transformado y desear de forma individual esta transformación que le permita: 
a. Concebirse y aceptarse como persona sujeta de derechos, lo cual implica reconocer y aceptar que tiene una serie de derechos que son parte de su ser integral y lo llevan a la lucha constante por estos, así como su defensa; este reconocimiento además le permite visualizar y reflexionar de forma crítica acerca de su propia realidad vinculada a estos derechos.

b. La misma concepción de sujeto de derechos conlleva a un reconocimiento individual de responsabilidades hacia sí mismo, de forma que le permitan defender y resguardar la realización de estos derechos, esta es la responsabilidad individual consigo mismo que tiene cada ser humano.

c. Vinculado a lo anterior se une el precepto del reconocimiento y aceptación de la corresponsabilidad social, que es la interiorización y la reflexión acerca de la responsabilidad individual que se tiene con otros, es llamada de forma general como los deberes que cada ser humano tiene y que se relacionan con los derechos. Sin embargo, esto no solo obedece a un principio social, sino que requiere de una aceptación, reflexión e interiorización interna de cada persona que le lleve a cuestionarse su rol en los espacios colectivos (familia, grupo, comunidad, sociedad) y las actitudes y acciones que esto conlleva.

d. A partir de estos tres elementos se puede hablar de un camino que va hacia la comprensión de la realidad política, económica, cultural y social y una visión crítica de las relaciones y situaciones que limitan la realización de los derechos humanos, o, por el contrario, permiten su vigencia plena. Esto es importante porque no se trata de presentar la educación en derechos humanos con una visión negativa de lo que acontece, sino de las situaciones positivas hacia un horizonte esperanzador.

Se puede hablar de que en el curso se abordan de manera más profunda los dos primeros, es decir, una educación en derechos humanos que cree en la transformación del ser humano que permita incidir en la colectividad, esto implica el trabajo con las actitudes y las acciones, el desarrollo de habilidades, la búsqueda de la reflexión acerca del papel del ser humano como individuo y como parte de una colectividad, en la cual sus acciones tienen importantes resultados. Esta no es una tarea fácil, puesto que salir de la zona de confort, de pasividad o de desilusión no se logra de la noche a la mañana. 
La alteridad va más allá de pensar en la otra persona e identificarme con sus logros, luchas y necesidades, es la comprensión, la solidaridad y las acciones que en realidad se pueden llevar a cabo y que permitan construir una sociedad más justa y de convivencia más armoniosa; se inscribe en una propuesta renovada en la que verdaderamente se le ofrece valor al otro, dándole nombre, cuerpo, y por sobre todas las cosas, palabra para que pueda expresar sus razones, pensamiento, argumentos (Vallejo, 2014, p. 120).

\section{El juego cooperativo que busca el acercamiento mutuo}

La afectividad que se trata como tema fundamental en la educación en derechos humanos se refiere a dos principios importantes: la afectividad hacia mí mismo(a) y la afectividad hacia quienes me rodean. Ambas son importantes de trabajar en el aula al iniciar el curso del cual se habla en este trabajo, esto implica el reconocimiento de las propias habilidades, capacidades y fortalezas y las de los compañeros y compañeras de grupo, además, permiten que se genere un acercamiento entre los miembros del grupo y un clima grupal de más confianza.

La afectividad da espacio, en primera instancia, para que cada persona pueda pensar y reflexionar en sí misma, de forma positiva, en cuáles son las habilidades, gustos, deseos y sueños, personas y circunstancias que les han permitido llegar hasta donde están hoy y reconocer quién es como ser humano y sujeto de derechos, es un espacio de afirmación individual.

En este sentido, se realizan juegos hacia el reconocimiento de habilidades, como la elaboración de un "Anuncio de sí mismo", este se trata de que el estudiantado elabore un anuncio de las habilidades, conocimientos y otras cualidades que describan a cada persona. No siempre es algo fácil para las personas porque requieren tiempo para pensar y reflexionar al respecto, además, porque no les resulta fácil expresar públicamente todo aquello que es positivo. Además, de que el juego les permite mirar de forma introspectiva, también les permite reafirmarse ante el grupo a través de un proceso comunicativo de expresión de quiénes son y cómo se asumen a sí mismos.

Además de sus conocimientos y carreras que estudian en sus anuncios, se destacan habilidades, valores y actitudes como las que se mencionan en la tabla 1:

En este aspecto resultan importantes las actividades que les permitan reflexionar acerca de los derechos humanos que son importantes, incluso aquellos que consideran derechos y no están establecidos en convenciones o declaraciones. 
URL: http://www.revistas.una.ac.cr/index.php/dialogo/index

CORREO ELECTRÓNICO: universidadendialogo@una.cr

DOI: https://doi.org/10.15359/udre.9-1.10

Tabla 1

Habilidades, actitudes y valores del estudiantado

\begin{tabular}{|l|l|l|}
\hline Habilidades & Actitudes & Valores \\
\hline Disciplina & Disfrute & Sinceridad \\
Escucha & Servicio & Honestidad \\
Detallista & Paciencia & Amabilidad \\
Dar consejos & Esfuerzo & Responsabilidad \\
Expresarse de forma directa & Buen sentido del humor & Puntualidad \\
Colaboración & Confiabilidad & Amor \\
Orden & Perseverancia & Amistad \\
Creatividad & Discreción & Tolerancia \\
Empatía & Independencia & Solidaridad \\
& Alegría & Respeto \\
& Solidaridad & \\
& Cariño & \\
& Comprensión & \\
& Gentileza & \\
\hline
\end{tabular}

Fuente: Elaboración propia basada en el juego “Anuncio de sí mismo”.

\section{Una piedra en el zapato}

El proceso de reconocer mis derechos es de gran importancia en la educación en derechos humanos, como ya se mencionó anteriormente, en estas actividades no se pretende jerarquizar los derechos, pero sí que el estudiantado reflexione acerca de que tiene derechos y que en algún momento estos han sido muy significativos, aunque sin darse cuenta no los haya valorado como tales o que reflexione acerca de un derecho humano en específico.

En este caso, en el curso se realiza una actividad con una pequeña piedra, se le dice al estudiantado que piense en un derecho que es muy significativo para cada uno de ellos, pero no se puede decir cuál es, se le da una piedra a cada uno y se les pide que la pinten de forma que esta represente ese derecho que es muy importante (puede tener símbolos importantes), la piedra debe caber en la mano, de manera que esta se pueda cerrar.

En esta etapa de juego se da un espacio de reconocimiento de los derechos de cada persona, lo que les permite reflexionar acerca de cuáles son estos derechos y cuáles se han aprehendido, es importante, además, pensar en las razones por las cuáles se considera que estos derechos son importantes para cada uno. Salen a relucir derechos como la libertad de expresión, el derecho a la vida, el derecho a la salud, derecho a una vivienda, derecho a tener una religión, a la igualdad. 
Revista Universidad EN DiÁlogo • Vol. 9, N. ${ }^{\circ}$ 1, Enero-Junio, 2019 • 159-170

ISSN 2215-2849 • EISSN: 2215-4752

URL: http://www.revistas.una.ac.cr/index.php/dialogo/index CORREO ELECTRÓNICO: universidadendialogo@una.cr DOI: https://doi.org/10.15359/udre.9-1.10

Se hace una ronda de juegos con la piedra, una de las cuales es en parejas, la consigna es que la pareja logre ver la piedra de su acompañante, y se dice que se vale todo. En este juego muchas de las personas lo ven como una competencia y ponen en práctica varias estrategias que van desde forzar a la persona para que les dé la piedra, forzarla a abrir la mano, hacerle cosquillas u formas de manipulación como ofrecer dinero. Incluso en el juego se denota cómo hasta se inventan el objetivo, que en ningún momento fue tomar la piedra, solo ver la de la pareja.

En esta etapa resulta importante retomar la importancia de la lucha que cada persona debe ejercer en la defensa de sus derechos humanos, y que esta no debe convertirse en una competencia, no implica un trabajo aislado, sino en colectividad, en sociedad, por la búsqueda de condiciones más justas y equitativas. Además, el punto de reflexión radica en la importancia de observar y reconocer cuáles son los derechos de las otras personas, de quienes están a mi alrededor. Este reconocimiento conlleva a ir trabajando hacia un compromiso que trascienda la preocupación y lucha individual.

En esta actividad se trabajan dos aspectos importantes, el primero es la reflexión personal acerca de un derecho que es trascendente en la vida de este estudiante, quizá haya varios, o no había tenido el espacio para pensar en esto. Además, asocian este con situaciones personales vividas, con oportunidades, vivencias o necesidades.

Por ejemplo, en uno de los grupos con los que se realizó esta actividad surgieron derechos en su mayoría que se refieren a la vida, la libertad, la vivienda, la alimentación, la libertad de expresión, la educación y la salud. Algunos de los comentarios al respecto se citan a continuación: "Es importante porque sin el acceso a una buena alimentación las personas no se pueden desarrollar de manera equilibrada y activa"; "esto lo veo como un derecho de ser uno mismo, ya que influye en nuestra forma de pensar [refiriéndose a la libertad de expresión]"; "porque pienso que para todas las personas lo primordial es estar saludable. Para mí es muy importante, tanto por la salud de mi madre como para seguir adelante sin ninguna enfermedad que impida mis sueños"; "porque para mí no vale vivir si uno no tiene una vivienda digna, alimentación decente y no tengo una oportunidad para autorrealizarme como persona".

Dado la diversidad de respuestas, se puede notar que la forma de pensar en mis derechos es diferente a la de los demás, esto representa una intrasubjetividad de lo que es o ha sido muy importante en la vida del estudiante con respecto a los derechos humanos, especialmente porque hacen alusión a vivencias en el marco de estos. 
URL: http://www.revistas.una.ac.cr/index.php/dialogo/index

CORREO ELECTRÓNICO: universidadendialogo@una.cr

DOI: https://doi.org/10.15359/udre.9-1.10

\section{Pensando en una convivencia pacífica: lo que yo puedo aportar para la paz}

Se realiza un juego llamado "La canasta de mi abuela", se trata de que el estudiantado en un círculo vaya pasando una canasta (caja o bolsa de acuerdo con la disponibilidad), cada vez que esta está en manos de una persona la consigna es decir "esta es la canasta de mi abuela y yo pongo...", cada persona dice un aporte que puede hacer para contribuir a la paz desde su propio espacio, así asumen un compromiso individual con respecto a un derecho en específico, en cuyo caso se escogió el derecho a la paz. Las propuestas del estudiantado se enfocan en las siguientes acciones y actitudes:

\section{Cuadro 1}

Aportes del estudiantado para contribuir a la paz

Evitar sentimientos de superioridad

Poner en práctica y promover la solidaridad y la amabilidad

El amor por y hacia uno mismo y los demás

Hacer afiches con lenguaje inclusivo

Denunciar

Promover la comunicación asertiva

Respetar

No ser indiferente ante la injusticia

Educar contra la violencia

Hablar de derechos humanos

Actitud de evitar violencia

No discriminar

Informarme e informar

Ser comunicativo

Ser respetuoso

Ser tolerante

No hacer sentir mal a las personas

Promover la convivencia y respeto entre los niños

Denunciar

Realizar talleres en instituciones educativas sobre educación Paz

Brindar talleres de género

Reconocer cuándo provoca violencia

Fuente: elaboración propia. 
Revista Universidad EN DiÁlogo • Vol. 9, N. ${ }^{\circ}$ 1, Enero-Junio, 2019 • 159-170

ISSN 2215-2849 • EISSN: 2215-4752

URL: http://www.revistas.una.ac.cr/index.php/dialogo/index CORREO ELECTRÓNICO: universidadendialogo@una.cr DOI: https://doi.org/10.15359/udre.9-1.10

En esta actividad es importante el reconocimiento que cada persona hace de sus capacidades y habilidades que le permitan incidir en la sociedad, a la vez se denota que permite una reflexión acerca de las corresponsabilidades que tiene el sujeto de derechos en la sociedad.

\section{Una visión compartida con la extensión universitaria}

Se considera que el vínculo entre la docencia y la extensión en la universidad es de importancia fundamental, no solo como apoyo a los proyectos, sino como parte de la incursión del estudiantado en la vida comunitaria y su relación con las situaciones sociales con las que se identifican los grupos. La extensión pretende acercar la academia a la sociedad para promover un aprendizaje mutuo y es una forma de incidir en la sociedad de forma positiva.

Además, la educación en derechos humanos es una labor que corresponde a la universidad en sus tres áreas, como lo son la extensión, la investigación y la docencia, ante esto la Declaración de México sobre Educación en Derechos Humanos en América Latina y el Caribe (2001) considera que la universidad debería incorporar los componentes de derechos humanos en sus carreras y desde una perspectiva amplia.

Ante esto la educación en derechos humanos es importante, en tanto cada estudiante tiene la posibilidad de verse a sí mismo como actor o actriz fundamental en la sociedad, con un rol representativo de la transformación de los contextos y aprender a ver las necesidades y fortalezas que tienen los grupos comunitarios de una manera crítica y sobretodo esperanzadora.

En este espacio, mediante las evaluaciones del curso (exámenes, como les llaman también), se logra establecer propuestas más allá del aula y trascender hacia lo comunitario, en grupos los y las estudiantes participan de forma creativa para generar propuestas para trabajar con grupos. Se han realizado afiches, los cuales han sido creados (no impresos) para compartir diferentes visiones de los derechos humanos, los afiches se han expuesto en la Biblioteca Central de la UNA, Joaquín García Monge, además se ha contado con la participación de estudiantes visitantes de un centro educativo de Heredia.

Otro de los componentes que vinculan el trabajo del curso con la extensión universitaria es la elaboración de propuestas para la educación en derechos humanos, esto lo realizan por medio de talleres dirigidos a grupos de primaria, las propuestas que realizan se logran después de haber trabajado la primera parte del curso por medio de la cual ya el estudiantado ha vivido el juego como 
experiencia y ha podido reflexionar acerca de la trascendencia de la educación en derechos humanos como eje para el individual y en la sociedad, además, ya han vivido un proceso de construcción significativa que les ha permitido interiorizar la vivencia de los derechos humanos en las relaciones humanas más allá de los componentes teóricos.

La elaboración de la propuesta lleva un proceso de construcción grupal y preparación de materiales para asistir al centro educativo y llevarlo a la práctica con el estudiantado de primaria. A partir de esta experiencia se edita una guía didáctica, la cual está conformada por todas las propuestas realizadas por los grupos que realizaron las actividades con el objetivo de que sea un insumo que se entrega al personal docente de la escuela que se visita para que así puedan multiplicar las experiencias en las aulas.

Con respecto a las experiencias que ha vivido el estudiantado, esta tiene mucho significado en lo referente a la visita al centro educativo, en especial porque muchos no han realizado actividades de extensión, vínculos con grupos comunitarios y en la mayoría de los casos tampoco con grupos de niños y niñas.

Estas actividades se vinculan con el proyecto de extensión Aula Activa: juegos cooperativos para la educación para la paz por medio del cual el estudiantado puede realizar giras académicas para trabajar con los grupos en las comunidades, en este caso se ha trabajado con grupos de una escuela de Heredia.

Este tipo de experiencias conlleva elementos importantes de preparación actitudinal, cognitiva y de praxis, lo cual no solo implica el trabajo en el curso, sino la preparación de los trabajos y materiales en horarios fuera de horario y toda la logística de transporte, negociación en la escuela para que las autoridades permitan el ingreso.

\section{Acerca de la praxis educativa y la experiencia}

En la educación en derechos humanos y en los procesos comunitarios resultan importantes los sentimientos que surgen de los encuentros en la praxis pedagógica, porque manifiestan las actitudes que ha generado el proceso en el estudiantado. Las y los estudiantes mencionan elementos que las y los motivaron a la realización de los talleres, más que ser parte del curso y de un proyecto en su esencia formal, las motivaciones muestran ser internas con respecto a la expectativa de los resultados e interacciones del proceso práctico, algunos de los comentarios lo expresan: "(...) disfrutar del momento"; "(...) si soy capaz de transmitir correctamente el mensaje y que ellos se sientan cómodos (...)". 
Revista Universidad EN DiÁlogo • Vol. 9, N. ${ }^{\circ}$ 1, Enero-Junio, 2019 • 159-170

ISSN 2215-2849 • EISSN: 2215-4752

URL: http://www.revistas.una.ac.cr/index.php/dialogo/index CORREO ELECTRÓNICO: universidadendialogo@una.cr

DOI: https://doi.org/10.15359/udre.9-1.10

En otros casos se refieren a la respuesta del estudiantado y al proceso propio de interacción con los niños y las niñas, así como el impacto en que esto podría resultar: "(...) tenía grandes deseos de conocer las perspectivas de los niños y las niñas de Guararí respecto de la aceptación de las diferencias (...)"; "(...) saber que ellos y ellas iban a aprender mucho del tema (...)"; "(..) observar su recepción del taller".

El proceso, además, permite una serie de aprendizajes para el estudiantado, los cuales manifiestan a través de sus palabras, lo cual es muy importante porque muchas veces se generan a través de un plan de trabajo una serie de objetivos para el grupo con el cual se trabajará en la comunidad, y se evalúan estos, pero, ¿qué es lo que aprendió el estudiantado como facilitador del proceso? Más allá de la elaboración de la propuesta, ¿cuáles fueron los aprendizajes individuales?, ¿cuáles fueron los aprendizajes al ser parte de un encuentro con un grupo?, ¿cuáles son los cuestionamientos en mi rol de educador(a) en derechos humanos?: "(...) estar conscientes de que generar un cambio está en nuestras manos, aunque sea en pequeña escala (...)"; "es necesario interactuar con ellos para conocerlos, escucharlos, poner atención a lo que dicen, mostrarles interés, ser afectuosos verbal y físicamente con cada uno, en la forma que a ellos les guste: una palmada en la espalda, una sacudida de pelo, chocar manos, etc."; "lo más importante que aprendí de esta experiencia es que hay muchísimo por trabajar y este trabajo debe empezar por uno, aunque sea pequeño el aporte que uno cree que da, para quienes lo reciben puede ser grande (...)".

Hay algunos elementos que mencionan en los aprendizajes que vale la pena destacar en sus roles de educadores(as) en derechos humanos, se visualizan como guías en los procesos asumidos, estos como participativos, en los cuales la niñez tiene el espacio para ser escuchada, algunos comentarios al respecto: "Aprendí acerca de lo importante que es acercarse a estos grupos de niños y niñas para guiarlos respecto a las diversas temáticas que se encuentran presentes en la sociedad (...)"; "aprendí (...) a ser solidaria cuando alguien necesita de ayuda (...)".

Además, se visualiza en sus expresiones la importancia de la motivación y el entusiasmo que ven en los niños y las niñas al momento de participar, siendo este un aspecto central en la educación en derechos humanos, en tanto, en esta es importante la participación de educadores entusiastas que generen esta misma reacción contagiosa en el estudiantado, pero que también es a 
URL: http://www.revistas.una.ac.cr/index.php/dialogo/index

CORREO ELECTRÓNICO: universidadendialogo@una.cr

DOI: https://doi.org/10.15359/udre.9-1.10

la inversa. En este caso se puede reflexionar acerca de que el aprendizaje en la educación en derechos humanos debe ser motivador, que enamore con su dinamismo y con su visión esperanzadora.

Otro aspecto importante fue el hecho de que aluden a que uno de sus aprendizajes a considerar son los pensamientos y sentimientos de los niños y las niñas, así como las diferentes perspectivas que tienen de los temas abordados. Esto es fundamental en la educación en derechos humanos, reconocer a la otra persona como sujeto que tiene una historia, conocimientos e ideas propias y no como un ser que solo recibe la información que le dan, es importante que quien educa reconozca el papel de las otras personas como sujetos participantes, pensantes, que tienen un espacio de participación y reflexión: “(...) tienen muchas ideas y formación, lo cual permitió ampliar más"; "escuchamos diferentes formas de ser por medio de la forma en que se comunicaban (...)"; "creo personalmente que uno aprende mucho (...)";

“aprendí (...) a conocer cuál es su pensamiento y sentir". Lo anterior muestra que quien educa en derechos humanos facilita procesos que permiten construir nuevas historias y esperanzas de forma colectiva y compartida.

\section{Reflexiones finales: hay caminos que parecen ser difíciles}

La educación superior no parece una tarea fácil para quien es docente, especialmente porque el estudiantado ha transitado una educación primaria y secundaria muy poco flexible e innovadora y me atrevería a decir que poco divertida, esto ha creado estructuras mentales muy verticales, cerradas y limitantes de las capacidades socioafectivas y creativas. Lo cual se refleja en la distancia que se da entre el estudiantado, los temores para disfrutar, crear e innovar. Lo anterior marca algunas particularidades que reflejan una educación que ha formado aprendientes que dirigen su camino a la búsqueda de un título universitario para lograr oportunidades laborales, situaciones que no solo se han notado en el estudiantado de nuevo ingreso, sino también en quienes van más avanzados en la carrera, claro que no se puede generalizar.

Estas complejidades que reflejan no solo las personalidades e historias de las personas, sino también la formación del estudiantado, llevan a preguntarse si la educación superior contribuye a reforzar estas estructuras a través de un verticalismo en la academia y si, además, la educación ha generado una búsqueda autómata de un espacio laboral más que un sendero hacia el aprendizaje para el desarrollo integral de cada ser humano. 


\section{Referencias bibliográficas}

Asamblea General de Naciones Unidas (2014). Plan de acción para la tercera etapa (2015-2019) del Programa Mundial para la Educación en Derechos Humanos. Informe de la Oficina del Alto Comisionado de las Naciones Unidas para los Derechos Humanos. Recuperado de https://documents-dds-ny.un.org/doc/UNDOC/GEN/G14/099/58/PDF/ G1409958.pdf?OpenElement

Vallejo, S. (2014). La pedagogía de la alteridad: un modo de habitar y comprender la experiencia educativa del presente. Revista Fundación Universitaria Luis Amigó, 1(2), 114-125. Recuperado de funlam.edu. co/revistas/index.php/RFunlam/article/download/1333/1210

Unesco, OHCHR (2001). Declaración de México sobre educación en derechos humanos en América Latina y el Caribe. Conferencia Regional sobre Educación en Derechos Humanos en América Latina y el Caribe. Recuperado de https://www.iidh.ed.cr/multic/UserFiles/ Biblioteca/IIDH/2_2010/AspecTeoMetodologico/Material_Educativo/ Declaracion-Mexico.htm 